\title{
Factores determinantes de los roles de filiales de empresas multinacionales: Caso Venezuela
}

\author{
Sigala Paparella, Luis E.* \\ León Darder, Fidel ${ }^{\star *}$ \\ Pla Barber, José ${ }^{\star \star \star}$
}

\section{Resumen}

Este trabajo estudia la influencia de los diferentes factores determinantes de los roles que tienen las filiales dentro de las empresas multinacionales, a través de la ponderación del comportamiento de factores del contexto de unas filiales sometidas a un entorno adverso. Se utiliza la metodología de estudio de casos en diecisiete filiales localizadas en Venezuela de diversos sectores. El estudio comprueba que la adversidad del entorno es uno de los factores contextuales que mayor influencia ejerce en la determinación de los roles de las filiales, induciendo a una devaluación de los roles de las mismas. El impacto de los determinantes varía dependiendo del tipo de filial: aquellas que realizan pocas actividades de valor agregado, usualmente de mercadeo y ventas, dependen más de la asignación de la matriz, mientras que las que incorporan un mayor número de actividades de la cadena de valor, integran otros determinantes como la adversidad del entorno y la iniciativa desarrollada por la propia filial.

Palabras clave: Filial exterior, matriz, rol, entorno, autonomía.

\section{Recibido: 24-02-06. Aceptado: 23-10-06}

Doctor en Dirección de Empresas, Universidad de València. Profesor Agregado, Universidad Centroccidental "Lisandro Alvarado"(UCLA), Barquisimeto, Venezuela.

E-mail: Isigala@ucla.edu.ve.

** Doctor en Ciencias Económicas, Profesor Titular de Escuela Universitaria, Departamento de Dirección de Empresas "Juan José Renau Piqueras", Facultad de Economía, Universidad de València, España. E-mail: Fidel.Leon@uv.es.

*** Doctor en Ciencias Económicas, Profesor Titular de Universidad, Departamento de Dirección de Empresas "Juan José Renau Piqueras", Facultad de Economía, Universidad de València, España. E-mail: Jose.Pla@uv.es. 


\section{Determining Factors in Multinational Corporation Affiliates: the Venezuelan Case}

\section{Abstract}

This article studies the influence of different determining factors in subsidiary roles within multinational companies, through determination of behavioral factors in the context of affiliated companies functioning under adverse conditions. A case study methodology is used in seventeen subsidiaries located in Venezuela with activities in different sub-sectors. The study shows that hostile environments are one of the factors that exert great influence on subsidiary role mandate determination, inducing a devaluation of the roles of the same. Overall impact of mandate determinants varies according with the subsidiary typology: those with a low value added activities, usually marketing and sales, essentially depends on company headquarter assignments, while subsidiaries with a larger number of activities in the value chain also integrate other determinants such as adverse environment determinism and subsidiary initiative developed locally.

Key words: External filial, headquarters, roles, environment, autonomy.

\section{Introducción}

Esta investigación estudia la influencia de los determinantes del rol de las filiales de empresas multinacionales sometidas a un entorno adverso, a través del análisis del comportamiento de los factores del contexto de filiales. El rol es el conjunto de actividades que realiza una filial dentro de la multinacional a la que pertenece para las que tiene reconocida responsabilidad (Galunic y Eisenhardt, 1996). Así, cambios en el número de actividades y responsabilidades implican una modificación del rol de la filial.

La literatura ha establecido que la determinación del rol de las filiales dentro de las multinacionales puede estar impulsada por la matriz, por el entorno del país de destino o por la iniciativa de la filial. Los autores (Birkinshaw y Hood, 2000; Frost et al., 2002; Birkinshaw et al., 1998), sin embargo, reconocen que el impacto del entorno como determinante del rol de la filial no ha sido tratado con suficiente pro- fundidad, tanto porque los trabajos se han desarrollado con base en el supuesto de que las filiales se encuentran en entornos locales estables, porque están referidos a un grupo de países desarrollados de pequeña y mediana dimensión.

Considerando que las teorías propuestas para países desarrollados pueden no ser las apropiadas para economías emergentes como Venezuela (Hoskisson et al., 2000), este trabajo pretende estudiar la influencia del entorno sobre la determinación del rol de las filiales.

El contenido del artículo se estructura en cuatro partes. En la primera parte se desarrolló el marco teórico sobre la integración de los determinantes del rol de las filiales y su relación con los factores del contexto organizativo de la filial. En esta sección también se presentan las proposiciones que fueron constatadas en el marco de este trabajo. La segunda sección describe la metodología de investigación y el protocolo que fue seguido para conducir el estudio. En la tercera 
parte presenta el análisis de los resultados obtenidos en la investigación y se discuten las proposiciones formuladas. Finalmente, en la última sección del estudio, se exponen las conclusiones de la investigación y las posibles implicaciones para futuras investigaciones.

\section{Determinantes del rol de las filiales}

Desde que la literatura sobre la empresa transnacional observó que las filiales exteriores tienen diferentes alternativas de inserción en el conjunto de la multinacional, dependiendo de la especialización de su estrategia (Bartlett y Ghoshal, 1986, 1987, 1989; Hedlund, 1986), surge la posibilidad de clasificar los diferentes roles estratégicos mediante una variedad de tipologías, entre otras, las de White y Poynter (1984), Bartlett y Ghoshal (1986, 1989), Ghoshal y Nohria (1989), Jarillo y Martínez (1990), Roth y Morrison (1992), Birkinshaw y Morrison (1995), y Gupta y Govindarajan (1991a, 1991b), que estudian el alcance de mercado/producto de las filiales, la importancia estratégica que tienen para sus corporaciones, el nivel de ajuste al entorno, el grado de integración a la multinacional y el flujo de conocimientos entre la matriz y las filiales.

Una vez identificados los diferentes roles que podían ser desempeñados por las filiales, se inició el estudio de los mecanismos que conducen a definirlos. Los tres determinantes responsables de inducir el establecimiento del rol son: la asignación de la matriz, la iniciativa de la propia filial y el determinismo del entorno local (Birkinshaw y Hood, 1997, 1998a, 1998b).

El primero de estos mecanismos se refiere a la fijación de responsabilidades por parte de la matriz, por eso se le llama "asignación de la matriz". El segundo, la "iniciativa de la filial", implica que es la propia filial la que impulsa la adopción de un determinado rol, y recoge las decisiones tomadas por la dirección de la filial, dentro del ámbito de sus competencias, para definir por ella misma el papel que asume. Finalmente, el tercer factor, denominado "determinismo del entorno local", implica que el rol de la filial puede ser alterado en función de las oportunidades y limitaciones del mercado local. Los tres mecanismos interactúan de forma dinámica para determinar el rol de la filial. De este modo, el rol de la filial condiciona y está condicionado por las decisiones adoptadas por los directivos de la sede central, las decisiones de los directivos de la propia filial y las condiciones del entorno local, que conducen a redefinir el rol de la filial en un momento dado (Birkinshaw y Hood, 1997, 1998a; Hood y Taggart, 1999).

Los mecanismos de determinación del rol de las filiales encuentran en el contexto organizativo ${ }^{1}$ el medio para modificar los niveles de recursos y capacidades de las filiales que conducen finalmente a

Se define como un conjunto de mecanismos administrativos y sociales que influyen en el comportamiento de los actores en la organización, sobre los que los directivos corporativos tienen algún control (Birkinshaw, 1999). 
cambios en el rol desempeñado por la filial. En este sentido, en la asignación del rol desde la matriz participan los factores del contexto corporativo, que sirven para controlar y medir el desempeño de la filial a través de mecanismos formales y administrativos tales como sistemas administrativos y políticas de remuneración e incentivos a los directivos (Bower, 1970). El segundo determinante está directamente relacionado con el comportamiento de la filial, pues la iniciativa es clave para generar capacidades distintivas en la filial que le permitan conseguir un cambio de rol. En el caso del último determinante, se encuentra el contexto del entorno local, que ejerce influencia sobre las decisiones tomadas tanto por la dirección de la matriz como de la filial, respecto de las actividades emprendidas por la filial.

El modelo de contexto organizativo que se observa en el Diagrama 1, expone la interacción existente entre los diferen- tes contextos. Por un lado, se ubica el contexto corporativo en un nivel externo, que se aplicará a todas las filiales de la corporación a través de las políticas y directrices corporativas que fije la matriz; en el nivel interno, se puede encontrar el contexto de la filial, que también depende de la iniciativa de la propia filial; además, existe un contexto del entorno local que ejerce influencia sobre los otros dos contextos. De acuerdo con la literatura (Birkinshaw, 1999; Birkinshaw y Hood, 1998a; Ghoshal y Nohria, 1989), los contextos agrupan una serie de factores diferentes que afectan el comportamiento de una filial localizada en un determinado país de destino.

Así, el contexto corporativo plantea los siguientes factores: la credibilidad de la filial ante la matriz, la descentralización de la toma de decisiones y la aversión al riesgo de la matriz. Por su lado, los factores del contexto de la filial serían: las ca-

\section{Diagrama 1 \\ Modelo de Contexto Organizativo en Entornos Adversos}

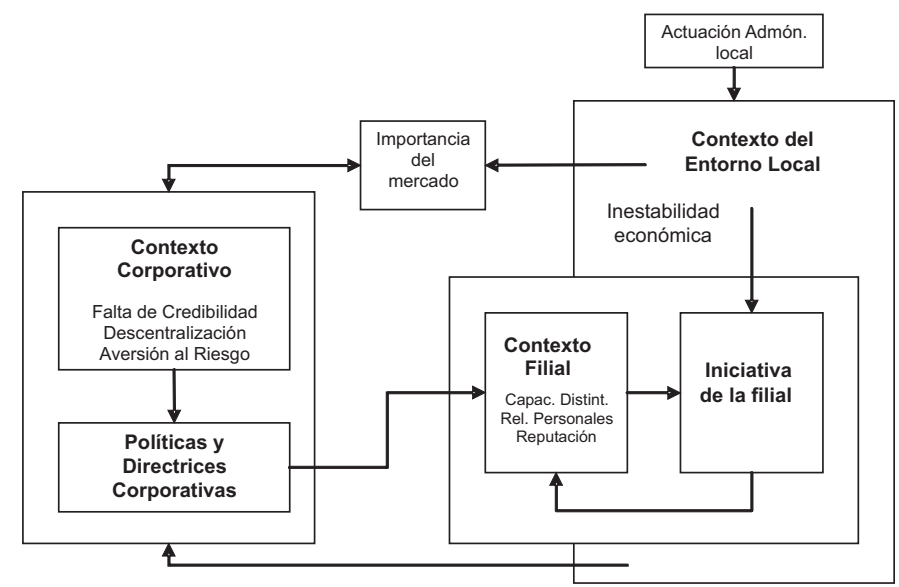

Fuente: Adaptado de Birkinshaw (1999). 
pacidades distintivas, la calidad de las relaciones personales entre directivos de la matriz y la filial, la reputación de la filial dentro de la corporación y, finalmente, su propia iniciativa. En tanto, los factores del contexto del entorno están representados por la inestabilidad económica, la importancia que tiene el mercado local para la multinacional y la actuación de la administración local.

Cada uno de esos factores contextuales contribuye a impulsar o devaluar el rol asignado a una filial exterior $y$, en algunos casos, el sentido puede cambiar dependiendo de las condiciones del entorno del país de destino que, en el caso de ser adverso, tenderá a inducir a una desvalorización del rol de las filiales. Seguidamente, se describirá la forma en que se espera que esos factores contextuales se comporten, y cómo se supone que ocurrirán los cambios en los mandatos de las filiales.

La credibilidad de la filial ante la matriz establece en qué medida los directivos corporativos son conscientes y confían en las capacidades y recursos de la subsidiaria local (Birkinshaw, 1997, 1999). En un entorno adverso, se puede presumir que el comportamiento de la matriz será de falta de credibilidad de la filial, lo que se traducirá en una fuerte resistencia (Birkinshaw, 1995a, 1995b, 1998; Birkinshaw y Fry, 1998) de la matriz ante las iniciativas y propuestas de la filial.

Por otro lado, un entorno adverso en un mercado extranjero puede despertar actitudes de aversión al riesgo, es decir, de baja tolerancia al riesgo, con la consecuente cautela, prudencia y resistencia respecto a decisiones que impliquen compromiso de recursos en el país de destino de la filial exterior.
Además, sí diferentes, nuevos o cambiantes entornos hacen más difícil la coordinación desde la matriz a través de la centralización, entonces se necesita una mayor descentralización en la toma de decisiones hacia las filiales que poseen un mejor conocimiento de los respectivos entornos locales (Garnier, 1982; Gates y Egelhoff, 1986).

Las filiales, igualmente establecerán una serie de estrategias para contrarrestar la resistencia de la matriz, orientadas a desarrollar capacidades distintivas, estrechar las relaciones personales con los directivos de la matriz, mejorar su reputación dentro de la corporación y emprender nuevas iniciativas.

Las capacidades y competencias distintivas le permiten a la filial generar un valor añadido superior al de otras filiales hermanas, mientras que la iniciativa predispone a los directivos de la filial a ser proactivos ante nuevas oportunidades para agregar valor a las actividades realizadas desde la filial (Birkinshaw, 1995a, 1995b, 1997, 1998; Birkinshaw y Fry, 1998; Birkinshaw y Hood, 1998a).

Se puede esperar que los directivos de las filiales situadas en ambientes adversos, en lugar de permitir la desvaloración de las filiales, desarrollarán capacidades que les permitan adaptar y gestionar las filiales para superar los obstáculos que le presenta el entorno; deberán ser proactivos tanto para adelantarse en el aprovechamiento de oportunidades y, como para responder hábilmente a los cambios de su entorno. El argumento se basa en que cada filial opera bajo un conjunto de condiciones diferentes al que se debe adaptar para ser eficaz, pues cada filial de empresa multinacional opera en 
un único entorno, que limita o determina sus actividades (Ghoshal y Bartlett, 1990; Ghoshal y Nohria, 1989; Rosenzweig y Singh, 1991). Adicionalmente, se debe pensar que estas filiales han sido exitosas en esa adaptación, puesto que se han mantenido en un entorno adverso y volátil durante un periodo bastante prolongado.

El establecimiento de relaciones personales con los directivos corporativos (Birkinshaw y Hood, 1998a; Birkinshaw, 1995b; Birkinshaw y Fry, 1998) permitirá a la filial tener participación política en la toma de decisiones de inversión y desinversión que la puedan afectar, además de influenciar en cierto grado el contexto corporativo.

La reputación de la filial dentro de la multinacional a la que pertenece, es uno de los parámetros críticos en contrarrestar la resistencia de la matriz ante las iniciativas de la filial, pues se basa la trayectoria de buen desempeño demostrada por la filial, como resultado de su experiencia pasada al satisfacer o superar las expectativas de la corporación durante largos periodos (Delany, 1998; Crookell y Morrison, 1990; Birkinshaw, 1997, 1999).

El contexto del entorno local juega un papel muy importante porque puede actuar como impulsor o supresor de la iniciativa de la filial, dependiendo de las condiciones que ofrezca (Birkinshaw, 1999). Un mercado con inestabilidad económica plantea condiciones opuestas a las que se han prescrito para estimular el desarrollo económico local, para incentivar las inversiones extranjeras directas, para asignar roles a las filiales y para promover el desarrollo de una filial exterior (Young et al., 1994).
La importancia del mercado local será afectada por la adversidad del entorno y, aunque de momento no cambie su grado de consideración, eventualmente generará una tendencia hacia la disminución del rol de la filial al ir perdiendo atractivo para la corporación.

La calidad y extensión de la actuación de la administración local influye sobre el rol de la filial. Unas políticas públicas adecuadas pueden ayudar al desarrollo local (Young et al., 1994); estas medidas tendrán sus mejores resultados si las instituciones gubernamentales que las ejecutan tienen un entorno de estabilidad que apoye las actividades de negocios de las empresas y, además, el apoyo institucional en el país de destino puede influir la toma de decisiones de la matriz respecto a la localización de recursos en ese mercado (Birkinshaw y Hood, 1998a) o ayudar a las filiales locales a reforzar e incrementar sus capacidades (Hood y Taggart, 1999).

Entre los factores contextuales planteados, se espera que algunos apoyen la valorización del rol de las filiales, mientras que otros factores favorezcan la pérdida de actividades y responsabilidades en el rol de la filial, dependiendo también de cómo actúe la adversidad del entorno sobre ellos. En este sentido, se espera que los factores vinculados con la matriz y el entorno local faciliten la desvalorización del rol de la filial mientras que los factores asociados a la determinación del rol impulsado por la filial favorecerán una valorización del mismo. En consecuencia, se pueden formular las siguientes proposiciones: 


\begin{tabular}{|l|l|}
\hline Proposición 1a. & $\begin{array}{l}\text { Los determinantes del } \\
\text { rol de las filiales que es- } \\
\text { tán asociados a los fac- } \\
\text { tores contextuales cor- } \\
\text { porativos y del entorno } \\
\text { local impulsarán a la } \\
\text { desvalorización del rol } \\
\text { de la filial cuando el en- } \\
\text { torno es adverso. }\end{array}$ \\
\hline Proposición 1b. & $\begin{array}{l}\text { Los determinantes del } \\
\text { rol de las filiales que es- } \\
\text { tán asociados a los fac- } \\
\text { tores contextuales de la } \\
\text { filial impulsarán a la va- } \\
\text { lorización del rol de la fi- } \\
\text { lial cuando el entorno es } \\
\text { adverso. }\end{array}$ \\
\hline
\end{tabular}

Más aún, con la pluralidad de roles que puede adoptar una filial exterior, los diferentes entornos en los que están inmersas y la variedad de enfoques estratégicos empleados por las empresas multinacionales, se puede pensar que la ponderación de fuerzas entre las tres perspectivas no tienen porqué estar en igual proporción entre los diferentes tipos de filiales.

En este sentido, se puede pensar que las filiales que realizan pocas actividades de agregación de valor, usualmente de mercadeo y ventas, dependerán más de la asignación del rol desde la matriz, mientras que en las filiales que desempeñan roles que implican un mayor número de actividades de la cadena de valor, incorporarán también como determinantes a la adversidad del entorno y la iniciativa de la propia filial. Así, se pueden presentar las siguientes proposiciones:

\begin{tabular}{|l|l|}
\hline Proposición 2a. & $\begin{array}{l}\text { Las filiales que operan } \\
\text { en entornos adversos } \\
\text { realizando pocas activi- } \\
\text { dades de valor añadido } \\
\text { estarán preponderante- } \\
\text { mente sometidas a la } \\
\text { determinación del rol } \\
\text { desde la matriz. }\end{array}$ \\
\hline Proposición 2b. & $\begin{array}{l}\text { Las filiales que operan } \\
\text { en entornos adversos } \\
\text { realizando numerosas } \\
\text { actividades de valor } \\
\text { añadido estarán pre- } \\
\text { ponderantemente so- } \\
\text { metidas a la determina- } \\
\text { ción del rol impulsado } \\
\text { por la propia filial y el } \\
\text { entorno local. }\end{array}$ \\
\hline
\end{tabular}

\subsection{Identificación de las filiales}

A fin de facilitar la identificación de las filiales exteriores que se encontraron en el estudio y su posterior análisis, se recurrió a la combinación de dos de las tipologías existentes en la literatura. En este sentido, se utilizó la clasificación de Jarillo y Martínez (1990), desarrollada a partir de las dimensiones grado de integración de las filiales exteriores a la empresa multinacional versus grado de localización, complementada con las dimensiones de la tipología de White y Poynter (1984), basada en el alcance de valor agregado de las actividades que realizan las filiales y el alcance de mercado que tienen.

Como se observa en el Cuadro 1 , se pueden encontrar filiales que desempeñan tres categorías de roles: las autónomas, centradas en el país de destino, llevan a cabo la mayoría de las actividades de la cadena de valor, de tal forma 


\section{Cuadro 1 Clasificación de las filiales}

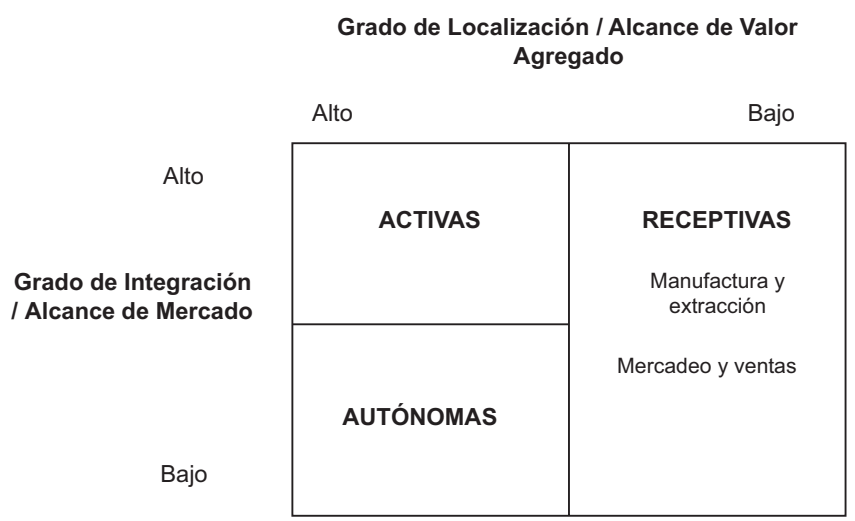

Fuente: Elaboración propia a partir de Jarillo y Martínez (1990) y White y Poynter (1984).

que son relativamente independiente de la matriz y de otras filiales (Jarillo y Martínez, 1990).

La filial adopta una estrategia receptiva si sólo unas pocas actividades de la cadena de valor son realizadas en el país de destino y están muy integradas con el resto de la empresa multinacional. Típicamente, se trata de las funciones de mercadeo y ventas, aunque también pueden ser puras operaciones de manufactura y extracción. La filial es activa, cuando realiza la mayor parte de las actividades de la cadena de valor en el país de acogida, y son realizadas en estrecha coordinación con el resto de la empresa, de tal forma que se juega un papel destacado como nodo activo dentro de una densa red interorganizativa (Jarillo y Martínez, 1990).

Las filiales autónomas se pueden asociar con las empresas multinacionales que presentan un enfoque estratégico multidoméstico, que compiten en sectores igualmente multidomésticos. Por otro lado, las filiales receptivas son propias de em- presas multinacionales globales que compiten en sectores globales, mientras que las activas son filiales que pertenecen a multinacionales que siguen un enfoque transnacional (Hout et al., 1982; Porter, 1986).

\section{Metodología de la investigación}

Para la comprobación empírica, se ha llevado a cabo una metodología cualitativa basada en el estudio de casos, y se comprobó que se reunían las condiciones que describen Yin $(1981,1994)$ y Eisenhardt (1989) para desarrollar una investigación de este tipo: permite entender mejor y con mayor profundidad cómo condicionan los entornos adversos a la determinación del rol de las filiales; no se tiene control sobre el fenómeno que se investiga, y los límites entre ese fenómeno y el contexto no están bien diferenciados.

Segun Yin (1994) y Rowley (2002), se sigue una estructura deductiva basada en la comprobación de unas proposicio- 
nes teóricas que se plantean en el marco teórico. Se debe señalar que este estudio tiene una limitación que se desprende de la metodología utilizada, pues el uso de los estudios de casos impide la generalización estadística (Yin, 1994). Sin embargo, si se puede indicar que los resultados y las conclusiones derivadas mantienen validez dentro de los límites señalados por las bases teóricas, que son pertinentes dentro de este marco (Bonache, 1999).

Se han estudiado múltiples casos como estrategia para abordar esta investigación y ha seleccionado Venezuela como país de destino, puesto que presenta un entorno que ha ofrecido evidencias $^{2}$ de adversidad creciente durante un periodo prolongado de tiempo.

Como unidad de análisis fueron utilizadas las filiales exteriores de empresas multinacionales, que se seleccionaron de acuerdo con los siguientes criterios: Debía tratarse de filiales exteriores de empresas multinacionales establecidas en Venezuela con actividades de producción o limitadas simplemente al mercadeo y ventas de productos elaborados en otras partes del mundo por la multinacional, y sólo se contempló aquellas filiales cuya propiedad estaba mayoritariamente (más de $50 \%$ ) en manos de corporaciones extranjeras.

La selección de los casos siguió criterios no probabilísticos con la intención de elegir filiales que tuvieran especial interés para la investigación. En ese sentido, las filiales tenían que reunir diferentes condiciones: 1) tener distintas dimensiones; 2) operar en diferentes sectores económicos; 3) tener asignados diferentes roles estratégicos; $y, 4)$ pertenecer a corporaciones multinacionales con diferentes enfoques estratégicos.

\subsection{Protocolo de la investigación}

La realización del trabajo de campo para este trabajo, se hizo durante el periodo comprendido entre los meses de diciembre de 2003 y enero de 2004. En ese tiempo, se hicieron varios contactos a diferentes filiales de empresas multinacionales que se ajustaran a los criterios de selección de los casos, que previamente se habían establecido.

La elección de los candidatos a ser entrevistados se realizó de acuerdo con un perfil que se estableció al inicio de la investigación. La persona a entrevistar debía estar al tanto de asuntos estratégicos de la filial, con conocimiento de sus operaciones internacionales de compras y ventas y sus relaciones con otras unidades de la corporación a la que perteneciera; además de, tener conocimiento de las operaciones de la subsidiaria en el mercado venezolano. Este perfil usualmente corresponde a cargos de alta dirección.

Finalmente, se debe comentar que se hicieron un total de diecisiete (17) entre-

2 Autores como Baptista (2003), Malavé (2002, 2003), Garay (2002, 2003), Márquez y Piñango (2003), Monteferrante (2002), Penfold (2002a, 2002b, 2002c, 2002d) y Sabal (2002) ofrecen mucha información sobre indicadores macroeconómicos, riesgo país y factores que elevan los costos de inversión en Venezuela que soportan esta afirmación. 
vistas para recabar información para igual número de filiales. De ellas, una fue realizada en la ciudad de Barquisimeto, otra en la ciudad de La Victoria, otra más en la ciudad de Valencia, y las restantes fueron realizadas en la ciudad de Caracas. Para guardar la confidencialidad de la información suministrada por las diferentes filiales, se usarán nombres ficticios derivados del alfabeto para hacer referencia a ellas.

\subsection{Fuentes de información}

Las entrevistas fueron la principal fuente de información. Como apoyo a esas entrevistas se utilizó un guión de preguntas, en su mayoría abiertas. Además, se solicitó las memorias de la empresa, informes anuales de la corporación o boletines internos, páginas web de las multinacionales y de las propias filiales y cualesquiera otros datos de archivo que pudiera suministrar la propia filial.

Adicionalmente, se recurrió a otros textos y documentos, como publicaciones locales especializadas, revistas de negocios, artículos de prensa local, entrevistas con antiguos empleados y bases de datos sobre corporaciones internacionales.

Con la finalidad de concretar y triangular la información, se elaboró un informe para cada filial exterior tomando como base la información suministrada en cada entrevista y todas las fuentes adicionales disponibles.

\section{Análisis de resultados}

\subsection{Características y roles estratégicos de las filiales}

La información utilizada en ésta investigación fue obtenida de 17 filiales ex- teriores de empresas multinacionales de diferentes tamaños, países de origen, sectores de actividad y enfoques estratégicos. Con relación al tamaño, el grupo de filiales estudiado pertenece a una variedad de multinacionales que va desde las más pequeñas con ventas anuales por valor de US $\$$ de 550 millones y una plantilla de 5.000 empleados, hasta aquellas que tienen ingresos por más de 50.000 millones de US\$ con nóminas de más de 80.000 empleados.

Como se observa en la Tabla 1, el origen de las corporaciones es igualmente variado. El conjunto está compuesto de siete multinacionales europeas de diversos países, siete norteamericanas y tres latinoamericanas. Los sectores de actividad son también variados, encontrándose distribuidas en industrias como: alimentos, bebidas y tabaco, cuidado personal, cosméticos, vinos y licores, tecnología, farmacéutico, equipos médicos, materiales para la construcción y porcelana sanitaria y dos de los sectores más importantes de la economía venezolana como lo son petróleo y gas, y aluminio. Alimentos, bebidas y tabaco tienen un perfil multidoméstico, cuidado personal está orientado hacia la transnacionalidad y el resto de los sectores se pueden calificar como globales (Kobrin, 1991).

De acuerdo con la información aportada por las filiales, los enfoques estratégicos seguidos por las corporaciones son igualmente variados: cinco son multinacionales globales, tres son multidomésticas y nueve son transnacionales. Por nacionalidades, de las americanas cuatro son transnacionales y tres son globales; en el caso de las latinoamericanas, dos siguen enfoques transnacionales $y$ 
Tabla 1

Datos de identificación de las filiales

\begin{tabular}{|c|c|c|c|c|c|c|}
\hline Filiales & Sector & $\begin{array}{l}\text { País de } \\
\text { Origen }\end{array}$ & $\begin{array}{l}\text { Ventas } \\
\text { US\$ MM }\end{array}$ & Nómina & $\begin{array}{c}\text { Año de } \\
\text { incorporación }\end{array}$ & $\begin{array}{c}\text { Forma de } \\
\text { incorporación }\end{array}$ \\
\hline A & Petróleo y gas & Europa & +100 & 450 & 2001 & Adquisición \\
\hline B & $\begin{array}{c}\text { Alimentos, } \\
\text { bebidas y tabaco }\end{array}$ & Europa & +100 & 100 & 50 's & Nvo. Establec. \\
\hline C & Tecnológico & EUA & +100 & 100 & 1993 & Nvo. Establec. \\
\hline D & Consumo masivo & Europa & $50-100$ & 350 & 60 's & Nvo. Establec. \\
\hline$E$ & Farmacéutico & EUA & $30-50$ & 200 & 1961 & Nvo. Establec. \\
\hline $\mathbf{F}$ & Tecnológico & EUA & $30-50$ & 150 & 1950 & Nvo. Establec. \\
\hline G & $\begin{array}{c}\text { Alimentos, } \\
\text { bebidas y tabaco }\end{array}$ & Europa & $30-50$ & $\sim 200$ & 1996 & Adquisición \\
\hline $\mathbf{H}$ & $\begin{array}{l}\text { Productos } \\
\text { Médicos }\end{array}$ & EUA & 20 & 100 & 1992 & Nvo. Establec. \\
\hline I & $\begin{array}{c}\text { Alimentos, } \\
\text { bebidas y tabaco }\end{array}$ & Europa & +100 & 650 & 1921 & Adquisición \\
\hline$J$ & $\begin{array}{c}\text { Alimentos, } \\
\text { bebidas y tabaco }\end{array}$ & LA & $\sim 250$ & 1550 & 1999 & Adquisición \\
\hline $\mathrm{K}$ & $\begin{array}{c}\text { Alimentos, } \\
\text { bebidas y tabaco }\end{array}$ & Europa & +100 & +1500 & 1995 & Adquisición \\
\hline $\mathbf{L}$ & $\begin{array}{c}\text { Alimentos, } \\
\text { bebidas y tabaco }\end{array}$ & Europa & +100 & $\sim 1000$ & 1953 & Nvo. Establec. \\
\hline $\mathbf{M}$ & $\begin{array}{l}\text { Materiales de } \\
\text { construcción }\end{array}$ & LA & 303,6 & $\sim 2200$ & 1994 & Adquisición \\
\hline $\mathbf{N}$ & $\begin{array}{c}\text { Alimentos, } \\
\text { bebidas y tabaco }\end{array}$ & EUA & +100 & 2100 & 60 's & Nvo. Establec. \\
\hline 0 & $\begin{array}{l}\text { Cuidado persnal. } \\
\text { y cosméticos }\end{array}$ & EUA & $10-30$ & 200 & 1958 & Nvo. Establec. \\
\hline $\mathbf{P}$ & Aluminio & EUA & 44 & 488 & 2000 & Adquisición \\
\hline $\mathbf{Q}$ & $\begin{array}{c}\text { Porcelana } \\
\text { Sanitaria }\end{array}$ & LA & 30 & 1030 & 1994 & Adquisición \\
\hline
\end{tabular}

Fuente: Elaboración propia.

una el multidoméstico, mientras que en el caso de las europeas, dos son multidomésticas, dos son globales y tres transnacionales.

En cuanto a las filiales exteriores de esas empresas multinacionales es- tablecidas en Venezuela, se pueden incluir algunas de las más importantes del país en sus correspondientes sectores, que varían, igualmente, en relación al tamaño y actividades desarrolladas. La más pequeña tiene ventas situadas en 
los 20 millones de US\$ con 100 empleados, mientras que las mayores alcanzan ingresos que sobrepasan los 300 millones de US\$ con plantillas de más de 2.200 empleados.

De ellas, ocho se dedican a actividades de limitado valor añadido, siete al mercadeo y ventas de productos manufacturados por sus corporaciones en otras partes del mundo, y una a actividades de exploración y explotación de petróleo y gas. Las nueve restantes realizan actividades con un amplio alcance de valor agregado, lo que implica que realizan actividades a lo largo de buena parte de la cadena de producción. Por otro lado, once de las diecisiete filiales se limitan a comercializar sus productos dentro de las fronteras del país de destino, mientras que únicamente seis alcanza mercados fuera de Venezuela.

De acuerdo con la clasificación de filiales que fue definida para este estudio, como se observa en la Tabla 2, en el grupo de filiales exteriores analizado se encuentran cinco filiales activas, cuatro filiales autónomas y ocho filiales receptivas, entre las que se pueden distinguir siete que realizan actividades de mercadeo y ventas y una que realiza actividades de extracción.

Las filiales con las plantillas menos numerosas son aquellas que responden a la tipología de receptivas de mercadeo y ventas, las cuales típicamente han sido establecidas en el país de nueva planta, producto del crecimiento orgánico de la corporación; en tanto que las filiales con nóminas más numerosas corresponden a filiales que desempeñan roles de autónoma o activa, donde es más frecuente la incorporación de filiales a la multinacional a través de una adquisición. En el caso de este subgrupo de filiales, se observa que todas, salvo una, son adquisiciones de reciente data.

La distribución de las filiales de acuerdo con el volumen de ingresos es más dispersa. Dentro del grupo de mayores ventas se sitúan por igual tanto aquellas que realizan sólo actividades de mercadeo y ventas (receptivas) como las que realizan actividades de producción con amplio valor agregado, tanto si tienen un elevado alcance de mercado (activas) como las que destinan sus ventas al mercado local (autónomas). Esto mismo sucede con el grupo de menores niveles de ventas.

En cuanto a la forma de incorporación, en el grupo de filiales se observan nueve establecimientos de nueva planta, en tanto que de las ocho adquisiciones, tres resultado de adquisiciones entre corporaciones y cinco fueron producto de la compra de empresas de propiedad local, distribuyéndose de la siguiente forma: tres compras fueron realizadas por corporaciones de origen europeo y dos fueron ejecutadas por multinacionales latinoamericanas.

La forma de incorporación de las filiales a las multinacionales varía según el origen de sus matrices. En el caso de las americanas, seis de los siete casos, la incorporación de las filiales fue por medio de establecimientos de nueva planta, en tanto que todas incorporaciones de filiales realizadas por las multinacionales latinoamericanas fueron a través de adquisiciones, mientras que las multinacionales europeas optaron por los establecimientos de nueva planta cuando hicieron sus primeras expansiones internacionales en las décadas de los 50's y 60's, mientras que en incorporaciones más recientes han preferido hacer adquisiciones. 


\section{Tabla 2}

Roles y enfoques estratégicos de las filiales

\begin{tabular}{cccccc}
\hline Filiales & Rol & $\begin{array}{c}\text { Enfoque } \\
\text { corporación }\end{array}$ & $\begin{array}{c}\text { Perfil } \\
\text { sectorial }\end{array}$ & $\begin{array}{c}\text { Alcance } \\
\text { de mercado }\end{array}$ & $\begin{array}{c}\text { Alcance } \\
\text { de valor }\end{array}$ \\
\hline A & Receptiva Extrac. & Global & Global & Limitado & Limitado \\
B & Receptiva M y V & Global & Global & Limitado & Limitado \\
C & Receptiva M y V & Global & Global & Amplio & Limitado \\
D & Receptiva M y V & Transnacional & Mult/Trans. & Limitado & Limitado \\
E & Receptiva M y V & Global & Global & Limitado & Limitado \\
F & Receptiva M y V & Global & Global & Limitado & Limitado \\
G & Receptiva M y V & Transnacional & Global & Limitado & Limitado \\
H & Receptiva M y V & Transnacional & Global & Limitado & Limitado \\
I & Autónoma & Transnacional & Multidom. & Limitado & Amplio \\
J & Autónoma & Multidom. & Multidom. & Limitado & Amplio \\
K & Autónoma & Multidom. & Multidom. & Limitado & Amplio \\
L & Autónoma & Multidom. & Multidom. & Limitado & Amplio \\
M & Activa & Transnacional & Global & Amplio & Amplio \\
N & Activa & Transnacional & Multidom. & Amplio & Amplio \\
O & Activa & Transnacional & Mult./Global & Amplio & Amplio \\
P & Activa & Transnacional & Global & Amplio & Amplio \\
Q & Activa & Transnacional & Multidom. & Amplio & Amplio \\
\hline
\end{tabular}

Fuente: Elaboración propia.

Adicionalmente, se puede indicar que las filiales europeas tienen mayor presencia en sectores que son preponderantemente multidomésticos y comparten con las norteamericanas su presencia en el global.

Con relación al comportamiento de las dimensiones alcance de mercado / alcance de valor agregado de las diferentes categorías de filiales, se puede señalar que, salvo por dos casos de filiales receptivas, se ajustan a las proporciones esperadas en cada rol: en las filiales que desempeñan un rol receptivo ambas dimensiones son limitadas; en las filiales que asumen un rol multidoméstico el alcance de mercado es limitado mientras que el alcance de valor agregado es amplio, en tanto que, en las filiales que desempeñan un rol activo ambas dimensiones tienen un amplio alcance.

En la Tabla 3, que se presenta a continuación, se resumen algunas de las características que más se destacan en el conjunto de filiales estudiado.

\subsection{Efectos del entorno en la determinación del rol de las filiales}

La percepción sobre la adversidad o no del entorno varía dentro del conjunto 


\section{Tabla 3}

\section{Resumen de las características de las filiales}

\begin{tabular}{lccc}
\hline Característica & \multicolumn{2}{c}{ Relación porcentual dentro del grupo de filiales } \\
\hline Roles de las filiales & $47 \%$ & $24 \%$ & 29 \\
& Receptivas & Autónomas & Activas \\
País de origen & $41 \%$ & $41 \%$ & $18 \%$ \\
& Europa & EUA & Latinoamérica \\
Perfil sectorial & $9 \%$ & $9 \%$ & $82 \%$ \\
& Multidoméstico & Trasnacional & Global \\
Enfoque de la corporación & $29 \%$ & $17 \%$ & $53 \%$ \\
& Global & Multidoméstico & Trasnacional \\
Forma de Incorporación & $47 \%$ & \multicolumn{2}{c}{$53 \%$} \\
Edad & Adquisiciones & Nuevos establecimientos \\
& $41 \%$ & \multicolumn{2}{c}{$59 \%$} \\
\hline
\end{tabular}

Fuente: Elaboración propia.

de filiales estudiado. En igual proporción un subgrupo considera que el entorno es adverso y otro subgrupo lo considera moderadamente adverso, mientras que existe un caso que percibe el entorno como favorable. Aún así, todas manifestaron por igual que sufren efectos negativos a causa de la adversidad del entorno, sin que apareciesen diferencias de su origen, tipo, tamaño o experiencia.

Más aún, cuando se les pide caracterizar el entorno por medio de una serie de variables previamente definidas ${ }^{3}$, coinciden mayoritariamente en señalar que los aspectos que más caracterizan el entorno venezolano son: la incertidumbre de las políticas económicas, la inestabilidad macroeconómica y la inseguridad ju- rídica y personal, como se puede observar en la Tabla 4.

Cada filial encuentra características y matices diferentes para describir el entorno venezolano, pero todas coinciden en que las condiciones se han tornado cada vez más desfavorables en el tiempo para sus actividades y negocios en el país. Ese desmejoramiento del ambiente de negocios ha generado, por un lado, mayores costos, que se han evidenciado en la necesidad de dedicar parte de sus recursos a realizar más actividades burocráticas y administrativas y en retrasos en la ejecución de planes e inversiones, y, por el otro lado, en una disminución de los niveles de ingresos, originado por una contracción generalizada del mercado.

3 Esas variables fueron definidas a partir de un estudio más amplio realizado por Penfold (2002cd). 


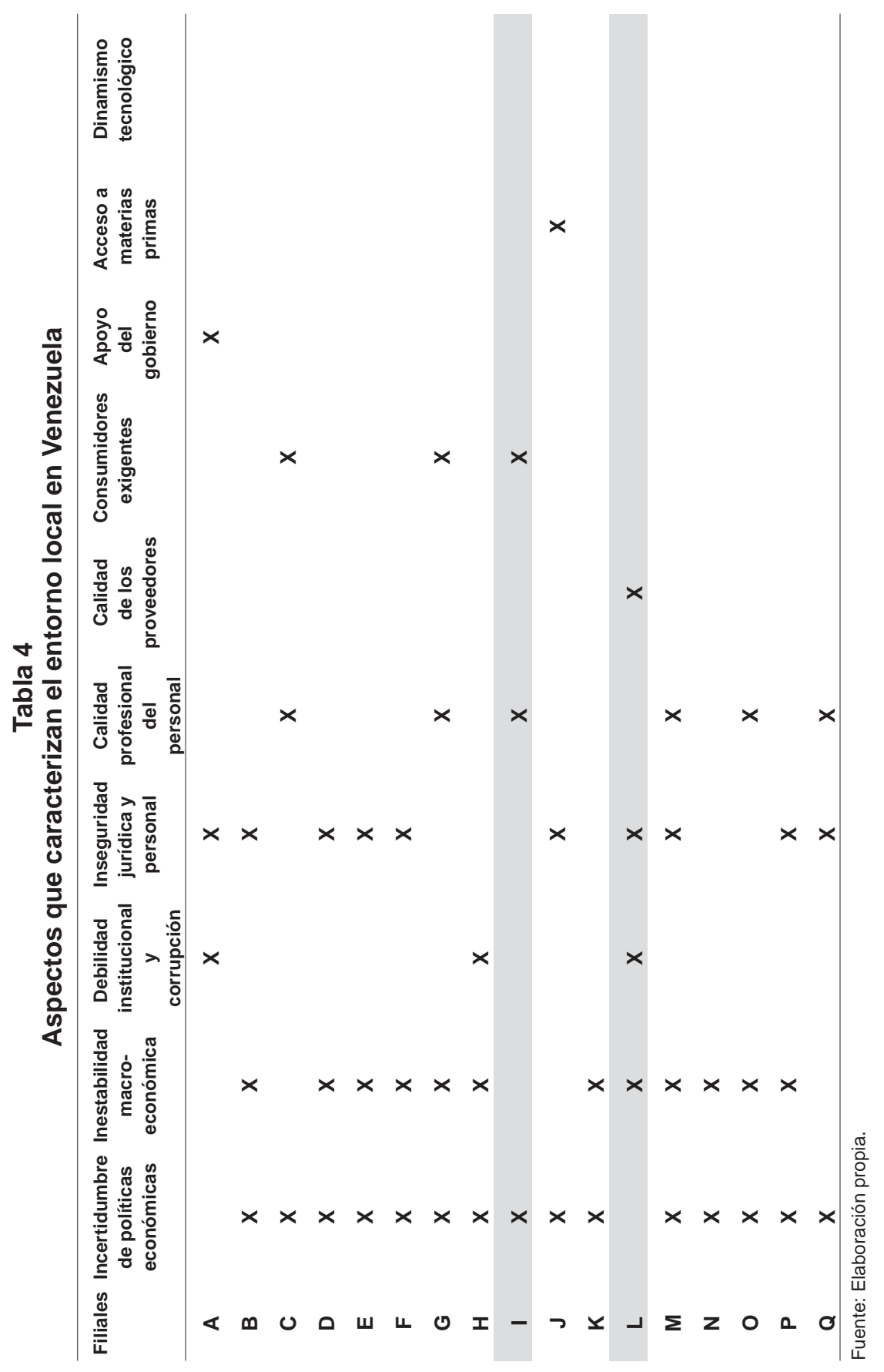


Aún así, en las condiciones político-económicas actuales de Venezuela, las filiales han encontrado situaciones que también las han favorecido como consecuencia de políticas aplicadas por el gobierno, como la disminución del número de competidores y el acceso a cuotas de importación en términos beneficiosos dentro de un mercado cambiario restringido. Por lo tanto, se puede pensar que, aunque la consecuencia general ha sido una disminución de los niveles históricos de rentabilidad, no han llegado a ser tan extremos como para que las respectivas multinacionales se planteen la salida del país.

Como aspecto positivo, pero en menor medida, las filiales estudiadas también han encontrado características favorables en el país de destino; se puede citar que seis de los casos indicaron que el entorno venezolano también ofrece calidad profesional de los recursos humanos y que tres filiales también indicaron que en el mercado venezolano existen consumidores exigentes en su sector (tecnológico y, alimentos, bebidas y tabaco).

Ahora bien, en el estudio se ha querido observar cómo es el comportamiento de un grupo de factores contextuales, tratando de indagar si la adversidad puede incidir en la determinación del rol que la filial pueda desempeñar, tanto en sentido positivo, bajo la posibilidad de que la filial pudiera mejorar su mandato como que, por el contrario, pudiera disminuir su rol dentro de la corporación a la que pertenece.

Ese comportamiento se puede observar en la Tabla 5 , donde se usan los símbolos "+" o "-" para indicar el sentido en que los factores afectarían a un cambio de mandato, o las siglas N/A, que implica que la filial considera que el factor no aplica como variable que influye en un cambio de su mandato.

Esto permite apreciar, en primer término, que el impacto que tienen los factores contextuales definidos en el marco teórico sobre la determinación del rol de las filiales se orientan de la siguiente forma: con sentido positivo, la "descentralización de decisiones de la matriz" dentro de los factores del contexto corporativo, los cuatro factores del contexto de la filial a saber: la "iniciativa de la filial", las "capacidades distintivas de la filial", la "reputación de la filial" y las "relaciones personales entre los directivos", y dentro del contexto del entorno, el "apoyo gubernamental" y la "importancia del mercado local". Respecto a este último factor, se debe aclarar que en el largo plazo, si las condiciones de adversidad de mantienen y continua reduciéndose el tamaño del mercado local, el efecto que producirá sobre el rol desempeñado por las filiales será negativo.

Por el otro lado, tienen impacto negativo sobre el rol desempeñado por las filiales, los factores del contexto corporativo "falta de credibilidad de la filial ante la matriz" y "aversión al riesgo de la matriz", y el factor del contexto del entorno "inestabilidad económica". En resumen, los resultados se expresan en el mismo sentido en que se realizó el planteamiento en el marco teórico, es decir, de los diez factores contextuales identificados, siete tienen sentido positivo y tenderán a favorecer el desarrollo y mejoramiento del rol de las filiales; en cambio, los tres restantes tienen sentido negativo y su impacto sobre el rol de la filial tendrá como consecuencia el declive del rol o pérdida de mandato en las filiales. 


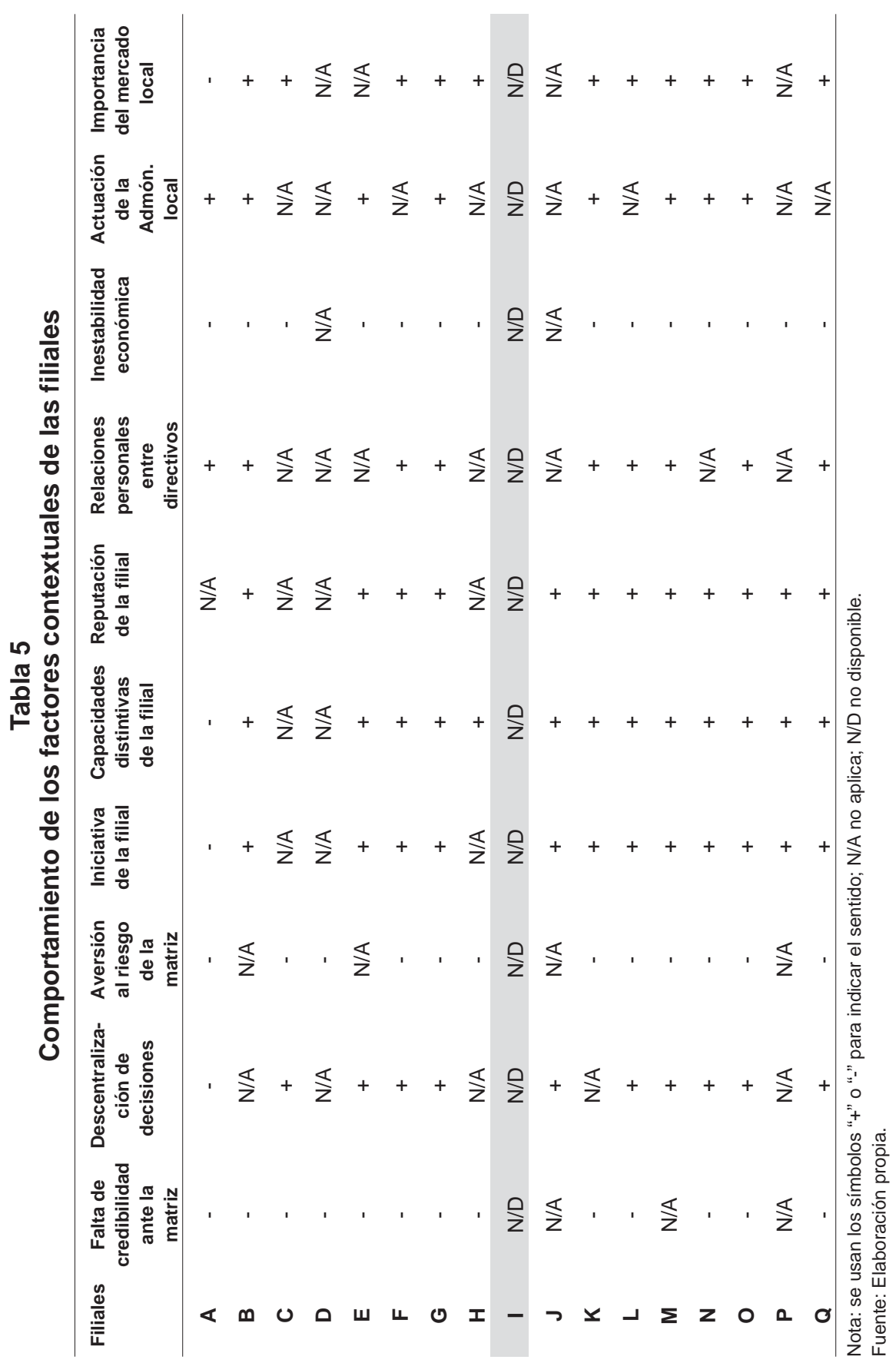


Además de conocer en qué sentido influyen los factores contextuales sobre la determinación del rol, también es posible determinar cuál es el grado de influencia que pueden tener, dependiendo del número de veces que hayan sido citados. En este sentido, se puede decir que los factores contextuales que mayor impacto ejercen en la determinación del rol del grupo de filiales estudiadas son la "inestabilidad económica" y las "capacidades distintivas de la filial", seguidas por la "falta de credibilidad ante la matriz" y la "iniciativa de la filial".

Esto implica que ante dos factores que afectan negativamente la determinación del rol, pues favorecen el declive o la desvalorización del rol, como serían la "inestabilidad económica" y la "falta de credibilidad ante la matriz", se contraponen otros dos factores que buscan el mejoramiento y desarrollo del rol de las filiales, como lo son las "capacidades distintivas de la filial" y la "iniciativa de la filial". Observando la fuente contextual de los factores en comento, se evidencia que mientras la corporación y el entorno favorecen el declive del rol, los factores de la filial lo hacen para desarrollar y mejorarlo.

Por otro lado, los factores contextuales que resultaron menos críticos para establecer el rol del grupo de filiales estudiadas son el "actuación de la administración local" y las "relaciones personales entre directivos" de la filial y la corporación, en virtud que han recibido el menor número de menciones $y$, por lo tanto, ejercen la menor influencia en la determinación del rol de las mismas.

Esto constata las dos primeras proposiciones. En el caso de la Proposición $1 \mathrm{a}$, si se observa el comportamiento con- junto de los tres factores contextuales corporativos se nota que los que más presión ejercen son aquellos que afectan el cambio de rol en sentido negativo, es decir, que impulsan la pérdida de roles. La "falta de credibilidad ante la matriz" y la "aversión al riesgo de la matriz" son más fuertes que la "descentralización de decisiones".

En tanto, en el grupo de factores del entorno, se observa que el más importante es la "inestabilidad económica", seguida de la "importancia del mercado local" $y$, a mayor distancia, el "actuación de la administración local". Aquí se verifica, igualmente, que el factor que más fuerza tiene afecta el cambio de rol en sentido negativo, a lo que se le debe agregar que, aunque la "importancia" actual del mercado local es todavía un elemento que favorece a las filiales, potencialmente generará una tendencia hacia la disminución del mandato de la filial al ir acumulando el efecto negativo de la adversidad sobre la economía local por un lapso más prolongado.

En el caso de la proposición 1b, como se indicó con anterioridad, los factores más resaltantes son las "capacidades distintivas de la filial" y la "iniciativa de la filial", seguidas por la "reputación de la filial". Estos factores, en conjunto, impulsan al mejoramiento del rol de la filial, de forma que se constata esta segunda proposición. El elemento "relaciones personales entre directivos" de la filial y la corporación tiene un peso moderado en este determinante.

Al revisar el impacto de los factores contextuales de acuerdo con las tipologías de filiales, se encuentran diferencias entre los factores que influyen sobre el subgrupo de filiales receptivas 
frente a los que lo hacen sobre el subgrupo conformado por las filiales autónomas y activas.

En el caso de las receptivas, se observa que los factores más citados son la "falta de credibilidad ante la matriz", la "inestabilidad económica" y la "aversión al riesgo de la matriz", por lo que existe una concentración en factores del contexto corporativo, articulados con la naturaleza adversa del entorno, mientras que los factores del contexto de la filial son los que menor influencia ejercen en este subgrupo de filiales. La tendencia que marca esta observación se traduce en que existe una fuerte presión hacia el declive o deterioro del rol que desempeñan el subgrupo de las filiales receptivas. Estos resultados confirman la proposición 2a.

Con relación a la proposición $2 \mathrm{~b}$, se observa que resultó constatada, por cuanto el subgrupo de las filiales que desempeñan roles activos o autónomos destaca porque los factores contextuales que mayor influencia ejercen son: la "iniciativa de la filial", las "capacidades distintivas de la filial", la "reputación de la filial" y la "inestabilidad económica". Los tres primeros factores pertenecen a la esfera de la filial y favorecen el desarrollo y mejoramiento del rol desempeñado por las filiales, mientras que en el caso del último factor, el efecto es contrario.

\section{Conclusiones}

Es significativo encontrar en este estudio que la mayor parte de las multinacionales que pertenecen a sectores considerados como globales, como es el caso de la industria de los productos médicos, materiales de construcción, cos- méticos y el aluminio, han adoptado un enfoque estratégico transnacional. Esta posición estratégica requiere que la corporación busque mecanismos para incrementar sus niveles de sensibilidad local en los mercados donde se encuentra presente. Esto seguramente ha contribuido a mejorar las posibilidades de supervivencia de sus filiales situadas en entornos adversos, como ha ocurrido en el caso de Venezuela.

Cuando se trata de tener inversiones en entornos adversos, el caso de Venezuela muestra que las multinacionales europeas y latinoamericanas son más tolerantes al riesgo que las americanas, porque mientras que las primeras incrementan los recursos y las actividades de las filiales que operan en el mercado venezolano, las americanas, en cambio, reducen sus recursos comprometidos al asignar a sus nuevas filiales actividades de poco valor agregado, limitadas al mercadeo y ventas de productos que la multinacional elabora en otros países.

Por otro lado, las recientes adquisiciones de empresas locales reflejan el interés de las corporaciones multinacionales en realizar economías de aprendizaje en los procesos de dirección y gestión de empresas sometidas a entornos adversos, lo cual les permite incorporar a sus activos intangibles un conjunto de capacidades y conocimientos que después pueden ser diseminados al resto de la multinacional, bien a través del intercambio de experiencias o de la movilidad de unos recursos humanos con elevada calidad profesional.

A pesar de una disminución de los niveles históricos de rentabilidad, por causa de mayores costos de inversión y 
producción, las filiales estudiadas siguen siendo exitosas. Algunas de ellas perciben que su entorno es moderadamente adverso e incluso una de ellas lo considera favorable, pero no dudan en afirmar que el ambiente de negocios en Venezuela se caracteriza fundamentalmente por la incertidumbre de las políticas económicas, la volatilidad macroeconómica y la inseguridad jurídica y personal.

Por otro lado, se pudo constatar empíricamente el sentido en que actúan los factores del contexto organizativo de las filiales en la determinación del rol de las filiales: siete de ellos influyen en sentido positivo y tres en sentido negativo. Así, se observa que, si bien los factores contextuales positivos están distribuidos entre el contexto corporativo, el de la filial y el del entorno, los factores que inducen a la pérdida de mandato y el declive del rol de las filiales, se ubican en el contexto corporativo ("falta de credibilidad ante la matriz" y "aversión al riesgo de la matriz") $y$ en el contexto del entorno ("inestabilidad económica").

Sin embargo, resulta significativo que, a pesar de la mayoría numérica de los factores favorables al desarrollo y mejora del rol de la filial, dos de los cuatro factores contextuales que mayor influencia ejercen en la determinación del rol de las filiales del grupo, sean de aquellos que actúan en detrimento del rol de las filiales, a saber: la "inestabilidad económica" y la "falta de credibilidad de la filial ante la matriz".

Como contrapartida a esta situación, las filiales estudiadas también destacaron las "capacidades distintivas de la filial" y la "iniciativa de la filial", como dos de los factores contextuales de mayor in- fluencia en el establecimiento del rol de las filiales del grupo que, seguidos en importancia por la "reputación de la filial", actúan a favor del desarrollo del rol de las filiales y dependen de la elección de la propia filial.

Adicionalmente, se observaron diferencias en el grado de importancia de los mecanismos determinantes del rol según el tipo de filial. Las filiales receptivas dependen más de las decisiones de las corporaciones para un cambio de mandato (asignación de la matriz), seguido por el determinismo del entorno, lo cual marca una tendencia hacia el declive o deterioro del rol que desempeñan. En cambio, en el caso de las filiales activas y autónomas, tienen mayor importancia para establecer el rol la "adversidad del entorno" $y$, los mecanismos asociados al contexto de la filial, de manera que si el primero actúa en detrimento del rol de las filiales, los factores del contexto de la filial lo hacen para mejorarlo.

Estos resultados son muy significativos si se les compara con los que pudieran ser obtenidos en entornos favorables, pues se puede suponer que, en principio, el único factor del contexto que se comportaría negativamente, alentando una pérdida de roles de las filiales, sería la falta de credibilidad de la matriz ante la filial, pero ya no por razones atribuibles a la adversidad del entorno como en este caso, sino a actitudes etnocéntricas de la matriz o por una mala reputación atribuible a la propia filial.

En consecuencia, en presencia de un entorno favorable se observarían un conjunto de factores contextuales con menor potencial de generar pérdidas de mandatos, en donde las filiales tendrían 
mayores facilidades para impulsar sus capacidades e iniciativas a fin de favorecer sus roles.

Finalmente, a pesar de las limitaciones que impone la metodología utilizada, se considera que el trabajo ofrece ideas interesantes para futuras investigaciones. Una de ellas sería orientarse a determinar el comportamiento de esos factores contextuales en entornos favorables, observando si existe la misma relación entre el tipo de filial y los diferentes mecanismos de determinación del rol de las filiales en entornos favorables $y$, si los determinantes del entorno guardan la misma ponderación que en los entornos adversos.

Otra idea sería dedicarse a estudiar cómo ha sido la evolución del rol de las filiales venezolanas, para determinar si efectivamente la orientación de los factores contextuales hacia una disminución del rol, es una tendencia que se ha manifestado con la pérdida de mandatos en las filiales venezolanas.

\section{Referencias Bibliográficas}

Baptista, Asdrúbal (2003). Las crisis económicas del siglo XX venezolano. En Márquez y Piñango: En esta Venezuela: realidades y nuevos caminos, 4768. Ediciones IESA, Caracas.

Bartlett, Christopher A. y Ghoshal, Sumantra (1986). Tap your subsidiaries for global reach. Harvard Business Review, November-December, Año 64, No. 6, pp 87-94.

Bartlett, Christopher A. y Ghoshal, Sumantra (1987). Managing Across Borders: New Organizational Responses. Sloan Management Review, Año 29, No. 1, pp 43-53.
Bartlett, Christopher A. y Ghoshal, Sumantra (1989). Managing across borders. The transnational solution, Harvard Business School Press, Boston.

Birkinshaw, Julian (1995a). Taking the initiative. Business Quarterly, Año 59, No. 4, pp 97-102.

Birkinshaw, Julian (1995b). Encouraging Entrepreneurial Activity in Multinational Corporations. Business Horizons, May-June, Año 38, No. 3, pp 32-38.

Birkinshaw, Julian (1997). Entrepreneurship in Multinational Corporations: The Characteristics of Subsidiary Initiatives. Strategic Management Journal, Año 18, No. 3, pp 207-229.

Birkinshaw, Julian (1998). Corporate Entrepreneurship in Network Organizations: How Subsidiary Initiative Drives Internal Market Efficiency. European Management Journal, Año 16, No. 3, pp 355-364.

Birkinshaw, Julian (1999). The Determinants and Consequences of Subsidiary Initiative in Multinational Corporations. Entrepreneurship Theory and Practice, Año 24, No. 1, pp 9-36.

Birkinshaw, Julian y Fry, Nick (1998). Subsidiary Initiatives to Develop New Markets. Sloan Management Review, Spring, Año 39, No. 3, pp 51-61.

Birkinshaw, Julian y Hood, Neil (1997). An Empirical Study of Development Processes in Foreign-owned Subsidiaries in Canada and Scotland. Management International Review, Año 37, No. 4, pp 339-364.

Birkinshaw, Julian y Hood, Neil (1998a). Multinational Subsidiary Evolution: Capability and Charter Change in ForeignOwned Subsidiary Companies. Academy of Management Review, Año 23, No. 4, pp 773-795.

Birkinshaw, Julian y Hood, Neil (1998b). Multinational corporate evolution and 
subsidiary development. Macmillan Press, London.

Birkinshaw, Julian y Hood, Neil (2000). Characteristics of Foreign Subsidiaries in Industry Clusters. Journal of International Business Studies, Año 31, No. 1, pp 141-154.

Birkinshaw, Julian, Hood, Neil y Jonsson, S. (1998). Building firm-specific advantages in multinational corporations: the role of subsidiary initiative. Strategic Management Journal, Año 19, No. 3, pp 221-241.

Birkinshaw, Julian y Morrison, Allen (1995). Configurations of strategy and structure in subsidiaries of multinational corporations. Journal of International Business Studies, Año 26, No. 4, pp 729-753.

Bonache, Jaime (1999). El estudio de casos como estrategia de construcción teórica: características, críticas y defensas. Cuadernos de Economía y Dirección de la Empresa, Enero-Junio, Año 2, No 3, España, CIVITAS, pp 123-140.

Bower, Joseph L. (1970). Managing the resource allocation process: a study of corporate planning and investment. Harvard Business School Press, Boston.

Crookell, Harold y Morrison, Allen (1990). Subsidiary Strategy in a Free Trade Environment. Business Quarterly, Año 55, No. 2, pp 33-39.

Delany, Ed (1998). Strategic Development of Multinational Subsidiaries in Ireland. En Birkinshaw y Hood: Multinational corporate evolution and subsidiary development, 239-267. Macmillan Press, London.

Eisenhardt, Kathleen M. (1989). Building Theories from Case Study Research. Academy of Management Review, Año 14, No. 4, pp 532-550.
Frost, Tony S., Birkinshaw, Julian M. y Ensign, P. C. (2002). Centers of Excellence in Multinational Corporations. Strategic Management Journal, Año 23, No. 11, pp 997-1018.

Galunic, D. Charles y Eisenhardt, Kathleen M. (1996). The Evolution of Intracorporate Domains Divisional Charter Losses in High-technology, Multidivisional Corporations. Organization Science, Año 7, No. 3, pp 255-282.

Garay, Urbi (2002), El riesgo país de Venezuela: ¿Qué significa? ¿Por qué es tan alto? Debates IESA, Año 7, No. 4, Venezuela, Ediciones IESA, pp 58-63.

Garay, Urbi (2003). La inestabilidad política y el riesgo país de Venezuela. En Márquez y Piñango: En esta Venezuela: realidades y nuevos caminos, 113124. Ediciones IESA, Caracas.

Garnier, Gerard H. (1982). Context and decision making autonomy in the foreign affiliates of U.S. multinational corporations. Academy of Management Journal, Año 25, No. 4, pp 893-908.

Gates, Stephen R. y Egelhoff, William G. (1986). Centralization in Headquarters-Subsidiary Relationships. Journal of International Business Studies, Año 17, No. 2, pp 71-92.

Ghoshal, Sumantra y Bartlett, Christopher A. (1990). The Multinational Corporation as an Interorganizational Network. Academy of Management Review, Año 15, No. 4, pp 603-625.

Ghoshal, Sumantra y Nohria, Nitin (1989). Internal Differentiation of the Determinants of Global Integration. Strategic Management Journal, Año 10, No. 4, pp 323-337.

Gupta, Anil y Govindarajan, Vijay (1991a). Knowledge flows and the structure of control within multinational corporations. Academy of Management Review, Año 16, No. 4, pp 768-792. 
Gupta, Anil y Govindarajan, Vijay (1991b). Knowledge Flow Patterns, Subsidiary Strategic Roles, and Strategic Control within MNCs. Academy of Management Proceedings, pp 21-25.

Hedlund, Gunnar (1986). The Hypermodern MNC-A Heterarchy? Human Resource Management, Año 25, No. 1, pp 9-35.

Hood, Neil y Taggart, James H. (1999). Subsidiary Development in German and Japanese Manufacturing Subsidiaries in the British Isles. Regional Studies, Año 33, No. 6, pp 513-528.

Hoskisson, Robert E., Eden, Lorraine, Lau, Chung Ming y Wright, Mike (2000). Strategy in Emerging Economies. Academy of Management Journal, Año 43, No. 3, pp 249-267.

Hout, Thomas, Porter, Michael E. y Rudden, Eileen (1982). How global companies win out. Harvard Business Review, September-October, Año 60, No. 5, pp 98-108.

Jarillo, J. Carlos y Martínez, Jon I. (1990). Different roles for subsidiaries: the case of multinational corporations in Spain. Strategic Management Journal, Año 11, No. 7, pp 501-512.

Kobrin, Stephen (1991). An Empirical Analysis of the Determinants of Global Integration. Strategic Management Journal, Año 12, Special Issue, pp 17-31.

Malavé, José (2002). Costos y obstáculos a los negocios: La opinión de empresarios y gerentes. Debates IESA, Año 8, No. 1, Venezuela, Ediciones IESA, pp 43-52.

Malavé, José (2003), Gerencia y negocios en la Venezuela de hoy: supervivencia y algo más. Debates IESA, Año 8, No. 4, Venezuela, Ediciones IESA, pp 32-39.

Márquez, Patricia y Piñango, Ramón (2003). En esta Venezuela: realidades y nuevos caminos. Ediciones IESA, Caracas.
Monteferrante, Patricia (2002). Los negocios: los otros dolientes de la inseguridad. Debates IESA, Año 8, No. 1, Venezuela, Ediciones IESA, pp 21-24.

Penfold, Michael (2002a). ¿Cuánto cuesta invertir en Venezuela? Debates IESA, Año 8, No. 1, Venezuela, Ediciones IESA, pp 8-11.

Penfold, Michael (2002b). Los costos de la debilidad institucional. Debates IESA, Año 8, No. 1, Venezuela, Ediciones IESA, pp 12-19.

Penfold, Michael (2002c). Costo Venezuela: Opciones de Política para Mejorar la Competitividad. Tomo I: Ensayos sobre reformas institucionales para disminuir costos de transacción. Consejo Nacional de Promoción de Inversiones, CONAPRI, y Corporación Andina de Fomento, CAF, Caracas.

Penfold, Michael (2002d). Costo Venezuela: Opciones de Política para Mejorar la Competitividad. Tomo II: Base Estadística sobre los costos administrativos a la inversión privada. Consejo Nacional de Promoción de Inversiones, CONAPRI, y Corporación Andina de Fomento, CAF, Caracas.

Porter, Michael E. (1986). Changing Patterns of International Competition. California Management Review, Año 28, No. 2, pp 9-40.

Rosenzweig, Philip M. y Singh, J.V. (1991). Organizational Environments and the Multinational Enterprise. Academy of Management Review, Año 16, No. 2, pp 340-361.

Roth, Kendall y Morrison, Allen (1992). Implementing global strategy: Characteristics of global subsidiary mandates. Journal of International Business Studies, Año 23, No. 4, pp 715-735.

Rowley, Jennifer (2002). Using Case Studies in Research. Management Research News, Año 25, No. 1, pp. 16-27. 
Sabal, Jaime (2002). Los riesgos del riesgo país. Debates IESA, Año 7, No. 2-3, Venezuela, Ediciones IESA, pp. 81-85.

White, Roderick E. y Poynter, Thomas A. (1984). Strategies for Foreign-Owned Subsidiaries in Canada. Business Quarterly, Año 49, summer, pp. 59-69.

Yin, Robert K. (1981). The Case Study Crisis: Some Answers. Administrative Science Quarterly, Año 26, No. 1, pp. 58-65.
Yin, Robert K. (1994). Case Study Research: Design and Methods. $2^{\mathrm{a}} \mathrm{Ed}$. Sage Publications, Thousand Oaks.

Young, Stephen, Hood, Neil y Peters, Ewen (1994). Multinationals Enterprises and Regional Economic Development. Regional Studies, Año 28, No. 7, pp. 657-677. 